Results End1/E6E7 HBD1 expression did not respond to stimulation. HBD1 secretion by Ect1/E6E7 cells more than doubled in response to IL-1 $\beta(p=0.0002)$ and IFN $\gamma(p=0.02)$, and was suppressed by progesterone $(p=0.02)$. End1/E6E7 HBD2 release almost doubled in response to LPS $(p=0.02)$ and progesterone $(p=0.01)$. HBD2 expression by Ect1/E6E7 cells doubled in response to LPS $(p=0.005)$ and halved after stimulation with progesterone $(p=0.006)$. End1/E6E7 HBD3 expression increased in response to LPS $(p=0.0009)$, and almost doubled after stimulation with PGN $(p=0.0003)$ and IFN $\gamma(p=0.027)$. End1/E6E7 HBD3 secretion almost tripled in response to incubation with progesterone $(p=0.003)$.

Conclusion Bacterial products elicited differential expression of HBDs in End1/E6E7 and Ect1/E6E7 cells. Progesterone mediated an increase in $\mathrm{HBD} 2$ and $\mathrm{HBD} 3$ secretion by endo-cervical cells but suppressed HBD1 and HBD2 secretion by ecto-cervical cells; this effect may augment cervical host defence, representing a novel mechanism by which progesterone may contribute to delaying the onset of preterm labour.

\section{PP.15 MORTALITY, CONGENITAL ANOMALY, \& MATERNAL RISK FACTORS ACROSS ETHNIC GROUPS IN BIRMINGHAM}

doi:10.1136/archdischild-2013-303966.296

${ }^{1} \mathrm{AM}$ Tonks, ' $\mathrm{A}$ Williamson, ${ }^{2} \mathrm{D}$ Willams, ${ }^{1} \mathrm{JO}$ Gardosi. 'Perinatal Institute, Birmingham, UK; 'West Midlands Regional Genetics Service, Birmingham, UK

Background Stillbirth and infant mortality rates in Birmingham remain consistently above those seen in the West Midlands and England \& Wales. Over half of the maternity population are from minority ethnic groups.

Methods Numerator data 2006-2010 (deaths/anomalies) were selected from the regional, population-based, anomaly and mortality registers, covering a birth cohort of 85,734. Denominator data for 2010, including ethnic group, consanguinity, and folate use, were available within the regional denominator database (www. pi.nhs.uk/peer/peerdata collection.htm).

Results Deaths from congenital anomaly comprise $29.3 \%$ of stillbirths and infant deaths in Birmingham. Mortality rates were significantly higher in Pakistani (odds ratio 3.0) and Bangladeshi mothers (odds ratio 2.1) compared to White Europeans. Pakistani mothers had significantly higher mortality rates from metabolic disorders, neural tube defects, and renal anomalies. In terms or primary and secondary screening, the prevalence of antenatal folate use was low in most minority ethnic groups. $49.4 \%$ of stillbirths and infant deaths had at least one anomaly that was amenable to detection by fetal anomaly screening programmes. However, in $5.1 \%$ of Birmingham births, the first booking appointment took place at 20 weeks or later. Pakistani mothers have the highest rates of consanguineous unions (49.9\%, CI 48.1-51.7, compared to $15.9 \%$ (CI 15.3-16) for all ethnic groups combined.

Conclusion Ethnic groups in Birmingham have an excess of perinatal mortality due to congenital anomalies. Interventions for these groups need to focus on improved folate uptake, timely access to screening services, and referral for genetic risk assessment.

\section{PP.16 PREGNANCY OUTCOMES IN WOMEN WITH KNOWN SICKLE CELL DISEASE: A CASE CONTROL STUDY FROM A DEDICATED TERTIARY JOINT OBSTETRIC SICKLE CELL CLINIC}

doi:10.1136/archdischild-2013-303966.297

'S Nanda, ${ }^{3} \mathrm{~S}$ Robinson, 'S Sankaran, 'P Kyle, ${ }^{2} \mathrm{~K}$ Nicolaides, 'E Oteng-ntim, A Weaver 'Women's Health, King's Health Partners, St Thomas' Hospital, London, UK; ' ${ }^{2 H a r r i s}$ Birthright Research Centre for Fetal Medicine, King's College Hospital, London, UK; ${ }^{3}$ Department of Haematology, Guy's and St Thomas' Hospital, London, UK
Introduction Sickle cell disease (SCD) in pregnancy has been associated with adverse pregnancy outcomes. However, with a multidisciplinary approach and surveillance, there is a suggestion of improvement in the overall outcome. The aim of this study was to evaluate the pregnancy outcomes in women with known SCD in a singleton pregnancy seen in our clinic, and to assess whether the presence of SCD alters the maternal serum analytes (Free B-human chorionic gonadotrophin and Pregnancy-associated plasma protein - A) at combined screening.

Methods Case-control study of all pregnant women with SCD undergoing combined screening from 01/01/2008 to 31/12/2011. Each case was matched with 3 non-SCD controls. Pregnancy outcomes in the two groups were compared.

\section{Abstract PP.16 Table}

\begin{tabular}{lll}
\hline Results & SCD $(\mathbf{n}=\mathbf{5 4})$ & Controls $(\mathbf{n}=\mathbf{1 6 5})$ \\
\hline Pre-eclampsia, n (\%) & $7(12.9)^{*}$ & $3(1.8)$ \\
Chronic hypertension, n (\%) & $1(1.8)$ & $4(2.4)$ \\
Live birth & $53(98.1)$ & $164(99.3)$ \\
CS, n (\%) & $20(37.7)$ & $42(25.6)$ \\
Vaginal, n (\%) & $33(62.3)^{*}$ & $122(74.4)$ \\
Gestation at delivery, wks, median (IQR) & $38(37-39)$ & $39(38-40)$ \\
Birth weight (grammes), median (IOR) & $3060^{*}$ & $\mathrm{AB3345}$ \\
B-HCG MoM, median (IOR) & $(2698-3280)$ & $(3000-3563)$ \\
PAPP-A MoM, median (IOR) & $1.2(0.65-1.85)^{*}$ & $1.05(0.62-1.63)$ \\
\hline
\end{tabular}

${ }^{*} \mathrm{P}<0.05$

Conclusion Women with SCD, when followed closely from the first trimester, have successful pregnancy outcomes. However, our data suggests an increased incidence of pre-eclampsia and lower birth weights compared to non-SCD controls. There was also a significant difference noted in the maternal serum analytes. Larger prospective studies are therefore required to assess the impact of SCD on combined screening.

\section{PP.17 PRE-GESTATIONAL DIABETES AND THE RISKS OF FETAL AND INFANT DEATH IN NORMALLY-FORMED OFFSPRING}

doi:10.1136/archdischild-2013-303966.298

'PWG Tennant, 'SV Glinianaia, ${ }^{2}$ RW Bilous, ${ }^{1,3} \mathrm{~J}$ Rankin, ${ }^{1,3} \mathrm{R}$ Bell. 'Institute of Health \& Society, Newcastle University, Newcastle-upon-Tyne, UK; ' James Cook University Hospital, South Tees NHS Trust, Middlesbrough, UK; ${ }^{3}$ Regional Maternity Survey Office, Newcastle-upon-Tyne, UK

Background Pre-gestational diabetes is associated with substantially increased risks of congenital anomalies, but the impact on normally-formed offspring is less well explored. This study explored the risks of fetal and infant death, examining the influence of $\mathrm{HbA1c}$, in normally-formed offspring of women with pregestational diabetes.

Methods All normally-formed singleton pregnancies in Northern England delivered during 1996-2008 were identified from the Northern Diabetes in Pregnancy Survey. Fetal ( $\geq 20$ weeks gestation) and infant deaths were identified from the Northern Perinatal Morbidity and Mortality Survey. Relative risks were estimated by comparing the prevalence rates between those with and without diabetes. The associations between peri-conception and third trimester $\mathrm{HbA1c}$ with each outcome were examined by logistic regression.

Results 400,158 normally-formed singletons were registered during the study period, including 1548 in women with pre-gestational diabetes. There were 46 fetal and 10 infant deaths following pregnancies in women with diabetes. 
The relative risks of fetal and infant death associated with pregestational diabetes were 4.54 (95\%CI: 3.41-6.05, p < 0.0001) and 1.82 (95\%CI: $0.98-3.38, p=0.06)$ respectively. The odds of a fetal or infant death increased by $19 \%(\mathrm{OR}=1.19,95 \% \mathrm{CI}: 1.02-1.39$, $\mathrm{p}=0.02)$ and $42 \%(\mathrm{OR}=1.42,95 \% \mathrm{CI}: 1.09-1.85, \mathrm{p}=0.01)$ respectively for each percentage increase in peri-conception $\mathrm{HbA} 1 \mathrm{c}$, although third trimester $\mathrm{HbA1c}$ was a stronger predictor of late fetal death $(\mathrm{OR}=1.67,95 \% \mathrm{CI}: 1.25-2.24, \mathrm{p}=0.001)$.

Conclusions Pre-gestational diabetes is associated with a substantially increased risk of fetal death in normally-formed offspring. The effect is largely moderated by glycaemic control, with increasing $\mathrm{HbA1c}$ conferring higher risks of both fetal and infant death.

\section{PP.18 CAESAREAN SECTION AND TIME TO NEXT BIRTH, ECTOPIC PREGNANCY, MISCARRIAGE OR STILLBIRTH-A DANISH REGISTER-BASED STUDY}

doi:10.1136/archdischild-2013-303966.299

${ }^{1} \mathrm{SM}$ O'Neill, ${ }^{3} \mathrm{E}$ Agerbo, ${ }^{2} \mathrm{LC}$ Kenny, ${ }^{\mathrm{T}} \mathrm{TB}$ Henriksen, ${ }^{5} \mathrm{PM}$ Kearney, 'RA Greene, ${ }^{3} \mathrm{~PB}$ Mortensen, ${ }^{1} \mathrm{JE}$ Lutomski, AS Khashan, ${ }^{2}$ S Meaney. ' National Perintal Epidemiology Centre, University College Cork, Cork, Ireland; ${ }^{2}$ Anu Research Centre, University College Cork, Cork, Ireland; ' National Centre for Register-based Research, Arrhus University, Aarhus, Denmark; ${ }^{4}$ Perinatal Epidemiological Research Unit, Arrhus University Hospital, Aarhus, Denmark; ${ }^{5}$ Department of Epidemiology and Public Health, University College Cork, Cork, Ireland

Aim Estimate time to next birth, risk of miscarriage, ectopic pregnancy or stillbirth in women with primary Caesareans.

Methods Danish population-registry cohort of births from 19822010 ( $n=833,162)$. Analysis using Cox-regression models.

Results Prior Caesarean group had longer birth intervals. No increased risk of ectopic pregnancy or miscarriage, but significantly increased risk of stillbirth among women with a prior Caesarean.

Conclusion Prior Caesareans were associated with an increased birth interval and increased risk of stillbirth compared to vaginal deliveries.

\section{Abstract PP.18 Table}

\begin{tabular}{lllll}
\hline & Birth Interval & Ectopic & Miscarriage & Stillbirth \\
\hline Delivery & adj.HR $(95 \% \mathrm{Cl})$ & & & \\
Vaginal & 1 & & & \\
Instrumental & $1.08(1.07,1.09)$ & $0.91(0.86,0.96)$ & $1.03(1.01,1.05)$ & $0.95(0.84,1.08)$ \\
Elective CS & $0.83(0.82,0.84)$ & $1.02(0.95,1.09)$ & $0.92(0.90,0.95)$ & $1.10(0.94,1.30)$ \\
Acute CS & $0.89(0.88,0.90)$ & $1.03(0.99,1.0)$ & $0.98(0.96,1.00)$ & $1.16(1.04,1.28)$ \\
MRCS & $0.64(0.60,0.69)$ & $1.04(0.69,1.57)$ & $0.78(0.66, .092)$ & $0.54(0.13,2.18)$ \\
\hline
\end{tabular}

Instrumental: vacuum/forceps; CS:Caesarean-section; MRCS: Maternally-Requested CS

\section{PP.19 WITHDRAWN BY AUTHOR}

\section{PP.20 THE POTENTIAL OF GENERAL PRACTISE DATA FOR CONGENITAL ANOMALY RESEARCH}

doi:10.1136/archdischild-2013-303966.300

R Sokal, K Fleming, LT Tata. University of Nottingham, Nottingham, UK

Background General practise data provide large population-based cohorts of individuals with prospectively collected medical information with promising potential for studying the causes and consequences of congenital anomalies. We sought to validate these data through comparison with congenital anomaly registries.

Methods Our study population was 794,209 children in The Health Improvement Network (THIN) primary care database, born between 1990 and 2009 with a median follow-up of 6.7 years. We compared the birth prevalence of any major and system-specific congenital anomalies with the European Surveillance of Congenital Anomalies (EUROCAT) United Kingdom registries.

Results The birth prevalence of any major congenital anomaly for children in THIN diagnosed before one year of age was 198 per $10,000(95 \%$ CI 195 - 201) which was slightly higher than the EUROCAT prevalence of 167 per 10,000 (Relative Risk 1.18, 95\% Confidence Interval $1.16-1.20)$. Absolute differences in prevalence between THIN and EUROCAT were small across 16 system-specific anomaly groups. The majority of children in THIN with major congenital anomalies had recorded diagnoses before 1 year of age (72\%), but including children diagnosed at any age increased the overall prevalence to 277 per 10,000 births.

Conclusions The prevalence of congenital anomalies in THIN was consistent with EUROCAT for early diagnoses, demonstrating THIN to be a valid source for investigating congenital anomalies. Age of diagnosis is an important factor in explaining a higher overall prevalence in THIN; the inclusion of diagnoses made after one year of age substantially improves capture of diagnoses making THIN more complete than registry data.

\section{PP.21 THE EFFECT OF FACTORS OF SOCIAL EXCLUSION ON ACCESS TO ANTENATAL CARE AND THE SUBSEQUENT IMPACT ON FETAL OUTCOMES}

doi:10.1136/archdischild-2013-303966.301

EM Mercer, DOC Anumba. University of Sheffield, Sheffield, UK

Background Delayed access to antenatal care is linked to maternal deaths and morbidity. Social deprivation also accounts for much maternal and child ill-health, with national health data showing discrepancies in the access and utilisation of care by groups of women who differ by ethnicity, age and socioeconomic status.

Objectives To map the prevalence of social deprivation and delayed access to antenatal care amongst women attending a regional referral maternity service and to correlate with fetal outcomes.

Methods Retrospectively analysed data collected from 59,847 singleton births at the Jessop Wing Hospital, Sheffield, UK, between 2002 and 2010, identifying maternal demographic features and neonatal outcomes. We plotted the Index of Multiple Deprivation (IMD) scores of women against the distribution of delayed access to antenatal care.

Results The geographic distribution of high deprivation scores and first pregnancy care attendance after 20 weeks gestation matched closely. High IMD scores increased the risk of being a late booker (OR: 1.092, 95\% CI: 1.01-1.18, $\mathrm{p}=0.031$ ) and being of minority ethnic extraction (OR: 5.6, 95\% CI: 5.2-5.9, p < 0.001), and significantly predicted low birth weight (OR: $1.66,95 \% \mathrm{CI}$ : 1.31-2.12, $\mathrm{p}<0.001$ ), premature delivery (OR:1.34, 95\% CI: 1.06 $1.70, p=0.017)$ and stillbirth (OR: $2.25,95 \% \mathrm{CI}: 1.68-3.01$, $\mathrm{p}<0.001)$. When sociodemographic variables were adjusted for, late booking did not independently predict adverse maternal or fetal outcomes.

Conclusions Disparities remain for women from certain backgrounds. Geographic mapping of high deprivation scores can direct research and health delivery strategies seeking to promote better access to pregnancy care and mitigate the resulting adverse fetal outcomes.

\section{PP.22 ANTEPARTUM HAEMORRHAGE OF UNKNOWN ORIGIN: SHOULD WE BE WORRIED?}

doi:10.1136/archdischild-2013-303966.302

S Bhattacharya, S Bhandari, EA Raja. University of Aberdeen, Aberdeen, UK 